\title{
Fondement de la persuasion et quête d'autorité chez Catherine de Sienne
}

Sonia Porzi

\section{CpenEdition}

\section{Journals}

Édition électronique

URL : http://journals.openedition.org/cei/252

DOI : $10.4000 /$ cei. 252

ISSN : 2260-779X

\section{Éditeur}

UGA Éditions/Université Grenoble Alpes

\section{Édition imprimée}

Date de publication : 15 avril 2005

Pagination : 63-88

ISBN : 978-2-84310-065-9

ISSN : 1770-9571

\section{Référence électronique}

Sonia Porzi, «Fondement de la persuasion et quête d'autorité chez Catherine de Sienne », Cahiers d'études italiennes [En ligne], 2 | 2005, mis en ligne le 15 octobre 2006, consulté le 27 mars 2021. URL http://journals.openedition.org/cei/252 ; DOI : https://doi.org/10.4000/cei.252 


\title{
FONDEMENT DE LA PERSUASION \\ ET QUÊTE D'AUTORITÉ \\ CHEZ CATHERINE DE SIENNE
}

\author{
Sonia Porzi \\ Université Paris 9
}

E perdonatemi, dolcissimo e santissimo Padre, che io vi dica queste parole. Confidomi che l'umiltà e la benignità vostra è contenta che elle vi sieno dette non avendole a schifo né a sdegno perché elle escano di bocca d'una vilissima femmina

écrit en 1379 au pape Urbain VI Catherine de Jacopo de Benincasa ${ }^{1}$, parfaitement consciente de l'incongruité qu'il peut y avoir pour une simple fille de teinturier à s'adresser à un pontife pour lui dicter sa conduite lors du grand schisme d'Occident. Face à lui en effet, comme face à son prédécesseur Grégoire XI, cette jeune fille "illettrée " (illetterata) de la petite bourgeoisie siennoise, restée qui plus est, fidèle à son état laïc, ne peut jouir a priori d'aucune forme d'autorité.

Qu'il s'agisse de convaincre les papes de rentrer à Rome, de réformer l'Église, de faire la paix avec les communes d'Italie centrale contre lesquelles les États pontificaux sont alors en guerre, ou de lancer la croisade, toute tentative de persuasion repose donc avant tout pour Catherine, sur une démarche de légitimation de sa propre parole.

Cette démarche, plus ou moins consciente, vise en fait pour elle à compenser son statut de femme, de laïque et d' "illettrée ", en fondant ailleurs son autorité : dans son action avant tout, et par-là dans sa renommée, c'est-à-dire dans l'image qui précède sa parole auprès du pape, mais aussi en plaçant son discours sous le couvert de tierces instances reconnues,

1. Santa Caterina da Siena, Le lettere, a cura di D.U. Meattini, Milan, Edizioni Paoline, 1987, lettre 370, p. 142 : «Et pardonnez-moi, très doux et très saint Père, si je vous dis ces mots. Je m’en remets à votre humilité et à votre bienveillance pour que vous acceptiez que ces mots vous soient dits, sans en ressentir mépris ni irritation, au motif qu'ils sortent de la bouche d'une très indigne femme ". C'est à l'édition citée que renverront les références aux Lettere. La numérotation des lettres adoptée par Meattini correspond à celle mise en place avant lui par Niccolò Tommaseo (CATERINA DA SiENA, Le lettere, ridotte a miglior lezione, e in ordine nuovo disposte, a cura di Niccolò Tommaseo, 4 vol., Florence, Barbera 1860). 
d'où elle tire son autorité : instances spirituelles ou humaines, ecclésiastiques ou laïques, mais dont la légitimité ne peut être remise en cause. En amont de son discours, Catherine revendique ainsi l'inspiration divine, tout en se plaçant sous la tutelle des auctoritates de la rhétorique sacrée. En aval, sa parole est constamment filtrée par des intermédiaires qui en contrôlent le fond et la forme, en présidant à la transcription puis à la diffusion de ses écrits.

Il apparaît ainsi que cette quête particulière d'autorité, qui confere à la démarche de persuasion catherinienne une dimension si originale, tient à la fois à des phénomènes textuels et extra-textuels dont on retrouve la trace aux niveaux socio-historique, philologique et littéraire.

\section{Le contrôle du confesseur}

À un premier niveau d'analyse, le contexte socio-historique dans lequel Catherine prend la parole, montre qu'elle s'inscrit dans une tradition qui mène progressivement les laïques à s'exprimer sur des questions religieuses de la plus haute importance, sous le contrôle de directeurs spirituels qui garantissent l'orthodoxie des propos tenus, et qui président ensuite à leur diffusion.

Écrites entre 1376 et $1380^{2}$, les vingt-trois lettres que Catherine adresse aux papes couvrent une période agitée de l'histoire de l'Église, allant des prémices de la guerre des Huit Saints aux premières années du grand schisme d'Occident. Le contexte politique de cette époque est particulièrement marqué par la question du siège de la curie pontificale.

Depuis près d'un siècle, l'influence politique de la papauté en Italie s'affaiblit progressivement, tandis que la prééminence française dans le gouvernement des États pontificaux s'accentue. Après la mort du pape Boniface VIII lors de l'attentat d'Anagni, perpétré contre lui par Philippe le Bel en 1303, son successeur Benoît XI tente en effet d'apaiser, pendant son bref pontificat, les querelles entre la papauté et le royaume de France. Mais le conflit ne cesse véritablement qu'avec l'élection du pape suivant, le Français Bertrand de Got, archevêque de Bordeaux. Elu en 1305, ce pape français, Clément V, refuse d'abord de se rendre à Rome pour son intronisation, et va ensuite s'installer définitivement en Avignon dès

2. Robert Fawtier, Sainte Catherine de Sienne, essai de critique des sources : les ceuvres de Catherine de Sienne, Paris, De Boccard, 1930, ch. XVI : "Essai de classement chronologique des lettres ", tableau récapitulatif, p. 310-317, et plus particulièrement p. 312-313. 
$1309^{3}$. Ainsi s'ouvre la période dite de la " captivité avignonnaise ", pendant laquelle, au fil de six pontificats successifs, la curie pontificale s'installe durablement en Avignon. Résultat de la pression exercée par Philippe le Bel pour l'élection d'un pape français, l'exil avignonnais apparaît aussi comme une fuite de la curie qui se réfugie en Avignon pour échapper à la situation de trouble qui règne dans les États pontificaux et à Rome en particulier, où les grandes familles (Orsini, Colonna, Caetani, etc.) s'affrontent pour le contrôle du siège pontifical et la domination des territoires environnants. Dès lors, la pacification des États pontificaux et la consolidation de l'autorité du pape dans cette zone apparaissent comme les préalables indispensables au retour de la curie à Rome.

Cet espoir, jamais vraiment abandonné pendant toute la période avignonnaise, se concrétise une première fois avec Urbain V en 1367. Mais son séjour romain est de courte durée. En effet, la même année, la mort du cardinal Albornoz, principal artisan de la reconquête des États pontificaux par l'Église, révèle la fragilité de cette pacification, de sorte qu'Urbain V retourne bientôt en Avignon où il meurt en $1370^{4}$.

Dès son élection, son successeur Grégoire XI fait part de son intention de reprendre à son compte le projet de ramener le Saint-Siège à Rome. Son retour, d'abord prévu pour septembre 1375, est sans cesse différé à cause des troubles qui s'intensifient dans les États du pape. À cette époque en effet, la tyrannie exercée par les légats pontificaux fait l'unanimité contre eux dans l'Italie du centre. En outre, leur politique expansionniste entre en concurrence avec celle des états voisins, Naples, Milan, mais surtout Florence qui s'engage alors dans une lutte ouverte contre le pape Grégoire XI. Ainsi éclate en 1375 entre les Florentins et les États pontificaux la guerre appelée "guerre des Huit Saints ". À l'instigation des Florentins, les villes et les états menacés se liguent contre le Pape : Sienne, Arezzo, Pise, Lucques, Viterbe rallient tour à tour la ligue, tout comme Bernabò Visconti bientôt rejoint par la reine Jeanne de Naples. Pour venir à bout de cette "ligue de la Liberté ", Grégoire XI devra recourir d'une part à l'interdit et à l'excommunication contre les Florentins en 1376, et d'autre part aux compagnies de mercenaires, les Anglais de John Hawkwood, les Bretons et les Gascons du cardinal Robert de Genève.

3. Bernard Guillemain, "Les cadres et les institutions de l'Église latine », in Histoire du Christianisme des origines à nos jours, éd. Jean-Marie Mayeur - Charles Pietri - André Vauchez - Marc Venard, XIV tomes, tome VI : Un temps d'épreuves (1274-1449), éd. Michel Mollat du Jourdin - André Vauchez, Paris, Desclée-Fayard, 1990, p. 45-48.

4. Jean-Pierre Delumeau - Isabelle Heullant-Donat, L'Italie au Moyen Âge, V'-XVe siècle, Paris, Hachette, 2000, p. 148, 186-187, 193. 
Lancées à l'assaut de la Romagne, des Marches et de l'Ombrie en 13761377, ces compagnies constitueront en fait l'avant-garde du Pontife effectivement de retour à Rome en janvier 1377. Si le Pape parvient à faire la paix avec Bologne, il meurt en 1378, avant de voir aboutir ses négociations de paix avec les Florentins.

Tandis que les Romains se répandent dans les rues au cri de « Romano lo volemo o almanco italiano ! ", les cardinaux élisent l'archevêque de Bari, Bartolomeo Prignani, sous le nom d'Urbain VI. Mais quelques mois plus tard, les circonstances qui avaient présidé à son élection, sont invoquées par certains cardinaux pour remettre en cause la légitimité du Pape. Réunis à Fondi, les cardinaux français procèdent à une nouvelle élection, désignant cette fois le cardinal Robert de Genève qui prend le nom de Clément VII, et part s'installer en Avignon dès 1379. Ainsi s'ouvre le grand schisme qui divisera la chrétienté jusqu'en 1417, les uns reconnaissant l'autorité d'Urbain VI (les urbanistes), les autres, comme le roi de France Charles V ou la reine Jeanne de Naples, reconnaissant celle de Clément VII (les clémentistes) ${ }^{5}$.

C'est à ces événements d'envergure sur lesquels s'affrontent les grands théologiens de l'époque, que Catherine va successivement prendre part, tant par l'action proprement dite, lors de voyages à Florence, Pise ou Avignon, où elle œuvre pour la réconciliation des villes toscanes avec le Pape, que par l'écriture, à travers ce que l'on peut appeler de véritables campagnes épistolaires menées pour soutenir UrbainVI dans le cadre du schisme $^{6}$.

Que Catherine Benincasa, "vilissima femmina » issue de la bourgeoisie siennoise, prenne la parole dans ce contexte pour s'exprimer sur des questions politiques, voilà certes une audace. Mais cette audace doit être considérée comme l'aboutissement d'une tradition qui a progressivement amené des femmes à s'adresser aux grands de ce monde pour les conseiller sur les choses publiques.

Au XII ${ }^{\mathrm{e}}$ siècle déjà, du fond du couvent de bénédictines dont elle est abbesse, Hildegarde de Bingen se permet de reprendre souverains et prélats, lançant ses avertissements jusqu'au pape Anastase IV lui-même. C'est alors en qualité de mystique visionnaire que cette aristocrate s'adresse au Pontife, sous le couvert de ses secrétaires et confesseurs, Volmar puis Gilbert de Gembloux ${ }^{7}$.

5. Ibid., p. 177-178, 193-195.

6. Pour une approche de ces lettres comme reflet de la propagande urbaniste, voir Antonio Volpato, "Le lettere di santa Caterina sullo scisma ", in La Roma di Santa Caterina da Siena, a cura di Maria Grazia Bianco, Rome, Edizioni Studium, 2001, p. 75-118.

7. Charmaine LEE, "Significato dell'autobiografia nel Medioevo ", in Manuale di letteratura 
Son exemple est suivi deux siècles plus tard par Brigitte de Suède, issue elle aussi de l'aristocratie (elle était apparentée à la famille royale), mais veuve et mère de plusieurs enfants, avant d'être fondatrice d'un ordre. En 1346, lorsqu'elle adresse au pape avignonnais Clément VI ses premières lettres de remontrances, elle mène une vie de prière et de pénitence aux abords du couvent cistercien d'Alvastra. Si elle lui rapporte alors que dans ses révélations, le Christ a qualifié le Pontife de "tueur d'âmes ", ses visions mystiques lui inspirent par la suite des conseils plus encourageants pour son successeur Innocent VI. "Mais ce n'est que sous Urbain V et Grégoire XI que Brigitte fit entendre une voix résolument vigoureuse pour la réforme de la papauté, le retour du siège pontifical à Rome et l'approbation de son ordre ${ }^{8}$. Il est difficile d'affirmer qu'elle détermine à elle seule Urbain $\mathrm{V}$ dans son projet de retour à Rome, mais ses rencontres répétées avec le Pontife semblent montrer que celui-ci ne méprise pas la parole de cette femme. Bien sûr, comme pour Hildegarde de Bingen avant elle, le crédit que peut avoir auprès du Pape Brigitte de Suède paraît encore lié pour une large part à ses origines aristocratiques. Mais avec elle, la prise de parole féminine dans le domaine public se déplace insensiblement d'un milieu strictement conventuel et aristocratique, vers un contexte plus laïc. Dans ce sens Brigitte de Suède anticipe véritablement Catherine Benincasa, à la fois issue d'une classe proprement bourgeoise et citadine cette fois, et fidèle toute sa vie à son état de laïque, puisque jamais elle ne rentrera dans les ordres. Ce mouvement de laïcisation de la prise de parole féminine dans le domaine spirituel s'inscrit en vérité dans le cadre plus vaste de l'émergence du rôle des laïcs dans l'Église, émergence elle-même liée à la revalorisation spirituelle de la vie active, longtemps dépréciée en faveur de la contemplation. Plus exactement, la prise de parole des femmes laïques apparait comme la toute dernière étape du processus qui, comme l'explique André Vauchez, conduit au fil du Moyen Âge, à « l'élargissement des catégories sociales appelées à jouer un rôle dans la vie ecclésiale " ${ }^{9}$. Cette prise de parole féminine s'effectue généralement sous la tutelle de clercs, comme c'est le cas pour Brigitte de Suède, dont les écrits sont vérifiés et diffusés par ses confesseurs, tel l'évêque espagnol Alphonse de Valdaterra qui fera la liaison entre Brigitte et Catherine ${ }^{10}$.

italiana. Storia per generi e problemi, a cura di Franco Brioschi e Costanzo Di Girolamo, vol. I : Dalle origini alla fine del Quattrocento, Turin, Bollati Boringhieri, 1993, p. 791-811, p. 808.

8. Margaret Wade-Labarge, "Brigitte de Suède (1303-1373) : mystique et prophète dans l'Église ", in Ne dormons plus, il est temps de se lever, Catherine de Sienne (1347-1380), éd. Elisabeth J. Lacelle, Paris, Cerf, 1998, p. 73-74.

9. André Vauchez, Les laïcs au Moyen Âge, Paris, Cerf, 1987, p. 3.

10. M. Wade-Labarge, "Brigitte de Suède... ", cit. p. 71, et Robert R. Fawtier - Louis Canet, La double expérience de Catherine Benincasa, Paris, Gallimard, 1948, p. 80. 
À la mort de Brigitte de Suède en 1373, Catherine a environ vingt-six ans et son activité épistolaire, inaugurée au moins dix ans plus tôt, prend de plus en plus d'ampleur, à mesure que sa renommée grandit ${ }^{11}$.

Selon ses hagiographes elle serait née à Sienne en $1347^{12}$ et son enfance aurait été très tôt marquée par des visions mystiques l'appelant à se rapprocher de l'ordre dominicain, à l'exemple d'un proche de la famille Benincasa, Tommaso della Fonte, qui deviendra bientôt son confesseur. Refusant le mariage auquel ses parents la destinent, elle obtient en 1363 la permission d'entrer dans le tiers ordre dominicain des Mantellate de Sienne malgré son jeune âge. Son statut de tertiaire lui permet de continuer à vivre dans la maison paternelle où une chambre lui est réservée afin qu'elle puisse, comme dans une cellule monacale, se retirer pour se consacrer à la prière.

Les plus anciennes lettres d'elle qui nous soient parvenues remontent à la moitié des années 1360, époque à laquelle elle s'engage résolument dans l'action. Aux côtés d'autres tertiaires plus âgées, veuves pour certaines d'entre elles, elle commence à assister les pauvres, les prisonniers, et les malades que la peste multiplie dans les rues de Sienne en 1374. C'est aussi le temps de ses premières actions de conversion, mais également de pacification dans cette ville que déchirent les luttes intestines entre l'ancien patriciat et la bourgeoisie. Ce véritable apostolat attire bientôt autour d'elle un groupe de disciples pour qui elle est la " mamma ".

Cette famiglia, constituée à la marge de l'ordre des frères prêcheurs, rassemble des hommes et des femmes d'horizons différents : des tertiaires, les Mantellate, mais aussi des clercs comme les dominicains Tommaso della Fonte ou Tommaso Caffarini, des franciscains, des augustins comme William Flete, des vallombrosains comme Giovanni delle Celle ou des Gésuates, et enfin des laïcs convertis par la "mamma ", tels le notaire Cristofano di Gano, l'homme de lettres Stefano Maconi ou le poète Neri di Landoccio Pagliaresi. Ces derniers, ainsi que d'autres membres instruits de la « lieta brigata ", prêteront d'ailleurs leur plume à Catherine dont ils deviennent les secrétaires, lorsque, au début des années 1370, son action politique commence à prendre de l'envergure.

11. Selon le classement chronologique des lettres par R. Fawtier (cf. supra note 2), la pièce la plus ancienne remonte à 1365-1366, mais il est vrai que rien n'exclut la composition de lettres avant cette date, qu'elles aient été ensuite perdues, ou qu’elles fassent partie du lot que R. Fawtier ne parvient à dater.

12. Concernant les polémiques sur sa date de naissance, voir Émile Jordan, «La date de naissance de Catherine de Sienne », in Analecta Bollandiana, XL, 1922. 
On ignore si Catherine a été personnellement en relation avec le Pape Urbain V, mais dès les années 1370 , elle entre en contact avec son successeur Grégoire XI par l'intermédiaire d'Alphonse de Valdaterra, ancien confesseur de Brigitte de Suède. À cette époque déjà, les premières lettres qu'elle adresse à des seigneurs, apparaissent comme le corollaire de son engagement aux côtés du Pape. En 1374, en butte à l'hostilité du franciscain Lazzarino da Pisa, elle se voit convoquée au chapitre général des dominicains à Florence ${ }^{13}$. C'est à l'issue de cette enquête, où son orthodoxie est confirmée, qu'on lui donne pour directeur spirituel Raymond de Capoue, son futur hagiographe ${ }^{14}$. Dès lors, sa correspondance s'intensifie tandis qu' elle multiplie les voyages en Toscane, pendant la guerre des Huit Saints, dans l'espoir de réconcilier le Pape avec les Florentins.

Dans les lettres qu'elle adresse à Grégoire XI dès 1376, ce dévouement sans limites s'assortit néanmoins des reproches les plus sévères au sujet du retour à Rome, continuellement différé : « Siatemi uomo virile ! » ${ }^{15}$, n’a-telle de cesse de lui répéter. Mais s'il est vrai que le retour effectif du Pape suit de quelques mois la visite que Catherine lui rend en Avignon, on s'accorde aujourd'hui pour relativiser l'influence qu'elle a pu exercer sur lui dans ces circonstances.

Après la mort de Grégoire XI, Catherine reste fidèle à son successeur Urbain VI qu'elle soutient au moment du schisme par une véritable campagne épistolaire destinée aux dissidents du camp clémentiste, tels le roi de France Charles V, la reine Jeanne de Naples ou les cardinaux italiens électeurs du nouveau Pape à Fondi. En 1379, l'appel lancé par Catherine à certains disciples pour qu'ils viennent la rejoindre à Rome afin d'entourer le Pape de leurs conseils, n'aboutit qu'en partie, et elle s'éteint, l'année suivante, laissant entre autres à son confesseur Raymond de Capoue, le soin de rassembler ses écrits et de les diffuser.

Il apparaît donc que la prise de parole de Catherine sur des questions politiques d'envergure est progressive, et qu'elle ne s'affirme vraiment qu'après 1374. Ce tournant semble en fait lié à deux facteurs qui conferent dès lors davantage d'autorité à son discours. D'une part, son action exemplaire lui a acquis une réputation de sainteté, comme l'atteste la

13. André Vauchez, «Sainte Brigitte de Suède et sainte Catherine de Sienne : mystique et prophétisme féminins aux derniers siècles du Moyen Âge ", in La vie spirituelle, $\mathrm{n}^{\circ}$ 640-641, septembredécembre 1980, p. 780.

14. Pour les références à cette œuvre hagiographique, connue sous le titre de Legenda Major, c'est à la traduction française de R.P. Hugueny que nous renverrons : RAYMON DE CAPOUE, Vie de sainte Catherine de Sienne, Paris, Tequi, 2002 [1904].

15. Lettre 206, p. 70 : «Montrez-vous un homme fort!» 
constitution autour d'elle d'un groupe grandissant de disciples qui reconnaissent son autorité. Dans ce sens, Catherine assoit son autorité sur le prestige qu'elle exerce auprès de ses adeptes, clercs et laïcs, dont certains jouissent personnellement d'une reconnaissance sociale, dans le domaine spirituel ou dans le cadre communal. Parmi eux, on compte en effet depuis 1367, un certain nombre de Gésuates, anciens disciples de Giovanni Colombini, une sorte de saint François siennois défendu par le pape Urbain $\mathrm{V}$ contre les suspicions d'hérésie qui pèsent sur ce marchand converti, ce "povero di Gesù Cristo " ${ }^{16}$. À sa mort, nombre de ses disciples confluent en effet vers la famiglia catherinienne, dessinant ainsi une sorte de filiation spirituelle entre Giovanni Colombini et Catherine ${ }^{17}$. On trouve aussi parmi les disciples de Catherine, des personnes reconnues dans la hiérarchie ecclésiastique, comme le dominicain Bartolomeo Dominici, bachelier à Pise dès 1374 , et lecteur à Florence, à Sainte Marie Nouvelle, l'année suivante, ainsi que des personnes influentes dans le cadre communal, comme le noble Matteo Cenni, que le sénat siennois nomme en 1374 Recteur de l'Hôpital de la Miséricorde. La confluence d'adeptes eux-mêmes reconnus socialement, contribue ainsi à consolider la renommée déjà acquise par Catherine à travers son action.

D'autre part, ce tournant dans l'investissement de Catherine en matière politique, semble lourdement déterminé par sa convocation au chapitre dominicain de 1374 d'où elle ressort, comme on l'a dit, avec une sorte d'investiture officielle. L'orthodoxie de ses propos ayant été confirmée, elle prend d'une certaine manière la succession spirituelle de Brigitte de Suède (morte l'année précédente) aux côtés de Grégoire XI, sous le contrôle du confesseur qui lui a alors été attribué. Comme l'a fait auparavant l'évêque Alphonse de Valdaterra pour Brigitte de Suède, Raymond légitime désormais, par son statut de dominicain et par l'autorité afférente à ce statut, le discours de Catherine qui, deux ans plus tard, se sent autorisée à écrire au Pape.

Comme d'autres mystiques du XIv ${ }^{\mathrm{e}}$ siècle, placées elles aussi sous la direction spirituelle de confesseurs, Catherine inverse bientôt cette relation d'autorité en sa faveur, faisant de Raymond de Capoue son porteparole auprès du $\operatorname{Pape}^{18}$. La plus ancienne des lettres de Catherine écrite à

16. Pour des éléments biographiques sur Giovanni Colombini, voir Giovanni Colombini, Le lettere, a cura di Dino Fantozzi, 2 vol., Lanciano, Carabba, 1924, vol. I, p. 21-26.

17. Sur l'identité des Gésuates que l'on retrouve dans la «famiglia » catherinienne, voir R. Fawtier - L. Canet, La double expérience..., cit., p. 74.

18. André Vauchez, "Le saint ", in L'homme médiéval, éd. Jacques Le Goff, Paris, Seuil, 1989, p. 363 : «Tout en se plaçant sous la direction de confesseurs ou de directeurs de conscience, elles [les 
Grégoire XI (lettre 185) fournit à cet égard un exemple intéressant. Outre les grands thèmes habituels, déjà évoqués, sur lesquels porte son discours (le retour à Rome, la croisade et la réforme de l'Église), on y trouve un conseil plus circonstanciel, lié à la réforme de l'ordre des frères prêcheurs, et plus précisément à la nomination d'un nouveau maître général, dans l'hypothèse où Élie de Toulouse, alors en charge, serait promu cardinal :

Ho inteso che' 1 maestro dell'ordine nostro voi 'l volete promuovere ad altro benefizio. Onde vi prego per l'amore di Cristo crocifisso, che, s'egli è così, che voi procuriate di darci uno buono e virtuoso vicario ; perocché l'Ordine ne ha bisogno, però che egli è troppo insalvatichito. Potretene ragionare con messer Nicola d'Osimo, e con l'archivescovo d'Otronto : e io ne scriverò a loro. ${ }^{19}$

Nous avons la chance de posséder les deux autres lettres (183 et 181) envoyées par Catherine à Jacques d'Itri, archevêque d'Otrante, et à Nicolas d'Osimo, protonotaire apostolique et secrétaire de Grégoire XI. Si les deux lettres reprennent globalement sur ce point la même formulation que la lettre adressée au Pape, on y trouve cependant une information supplémentaire, concernant l'identité de la personne que Catherine pressent pour remplacer Élie de Toulouse à la tête de l'ordre, mais qu'elle ne nomme pas expressément dans la lettre au Pape. Il s'agit d'Étienne de la Combe, dont elle tisse les louanges auprès de l'archevêque et du protonotaire apostolique ${ }^{20}$. Il apparaît donc clairement que ces deux destinataires sont chargés de suggérer au Pontife le nom d'Étienne de la Combe, et on peut se demander pourquoi Catherine choisit délibérément de ne pas le faire ellemême dans sa lettre au Pape ${ }^{21}$. En vérité, elle n'a pas directement voix au chapitre et son intervention en la matière peut paraître incongrue. Dans ce cas précis, sa suggestion ne semble avoir de place auprès du Saint-Père

laïques] ne tardèrent pas, bien souvent, à inverser à leur profit cette relation d'autorité, faisant d'eux leurs secrétaires et leurs porte-parole».

19. Lettre 185, p. 63 : «J'ai entendu dire à propos du responsable de notre ordre que vous voulez l'élever à un autre bénéfice ecclésiastique. C'est pourquoi, s'il en est ainsi, je vous prie, pour l'amour du Christ crucifié, de faire en sorte de nous donner un vicaire bon et vertueux, parce que l'ordre en a besoin, car il est trop sauvage. Vous pourrez en discuter avec messire Nicolas d'Osimo et avec l'archevêque d'Otrante ; et moi, je leur écrirai à ce sujet ".

20. Lettre 183 à Jacques d'Itri, p. 210 : «Vorrei che lo [il Papa] informasse di Maestro Stefano della Cumba che fu procuratore dell'Ordine della Provincia di Tolosa. Credo che se egli cel darà, sarà grand'onore di Dio e racconciamento dell'Ordine ; perocché mi pare ch'el sia uomo virile e virtuoso, e senza timore». Lettre 181 à Nicolas d'Osimo, p. 243 : «Pregovi che gli [al Papa] ragioniate, se vi pare, di Maestro Stefano, che fu Procuratore dell'Ordine quando frate Raimondo era in corte. Credo che sappiate ch'egli è uomo buono e virile. Spero che se noi l'avessimo, che per la grazia di Dio e per lui l'Ordine si racconcerebbe ».

21. Elle le précise en effet à ses deux destinataires : lettre 183 à Jacques d'Itri, p. 210 : « Io n’ho scritto al Padre santo : non ho detto però cui egli ci dia ; ma ho pregato che cel dia buono, e che ne ragioni con voi e con messer Nicola d'Osimo ", et lettre 181 à Nicolas d'Osimo, p. 243 : « Honne scritto al padre santo ; non pero detto cui egli ci dà, ma hollo pregato che cel dia buono, e ragionine con voi e con l'arcivescovo d'Otranto ». 
que si elle est avalisée par ces deux autorités ecclésiastiques. La fin des lettres que Catherine adresse à Jacques d'Itri et à Nicolas d'Osimo, montre qu'en vertu du même processus, c'est ensuite son confesseur Raymond de Capoue, qui est appelé à confirmer le bien fondé de cette suggestion vis à vis de l'archevêque et du protonotaire apostolique eux-mêmes ${ }^{22}$.

Cet exemple fait donc apparaitre que l'autorité dont peut se prévaloir Catherine quand elle s'adresse au Pape sur des questions de cet ordre, se consolide au fil d'une sorte de voie hiérarchique dont Raymond de Capoue est pour ainsi dire la pierre de touche.

Loin d'être propre à Catherine, ce processus de légitimation apparaît comme une constante chez de nombreuses mystiques médiévales souvent entourées de confesseurs, conseillers ou directeurs de conscience. À ces clercs qui garantissent l'orthodoxie de leur parole, fait généralement écho un autre type d'intermédiaire, le secrétaire, un lettré chargé quant à lui d'en assumer le passage à l'écrit, qu'il s'agisse seulement de transcrire ce qu'il entend, d'y apporter de simples corrections grammaticales, comme celles de Volmar sur les écrits d'Hildegarde de Bingen, ou d'en prendre en charge la traduction latine, comme le font successivement Pierre, prieur d'Alvastra et Alphonse de Valdaterra pour les visions rédigées en suédois par Brigitte de Suède ${ }^{23}$. Dans tous les cas, le travail du secrétaire semble lié à la nécessité de produire une œuvre écrite en latin, ce qui exige des compétences auxquelles les femmes n'ont pas accès à l'époque. Bien que s'agissant d'une production en langue vulgaire, les œuvres de Catherine présentent ce type de caractéristiques.

\section{La mise en forme par les secrétaires, disciples et éditeurs}

À un niveau philologique, l'histoire des textes catheriniens, de leur production à leur édition, révèle en effet la présence constante d'un intermédiaire entre Catherine et ses écrits, des premiers secrétaires aux éditeurs, en passant par les disciples qui prennent l'initiative de rassembler ses œuvres éparses, d'abord pour leur propre compte, puis dans une perspective plus large de vulgarisation de son message.

22. Lettre 183 à Jacques d'Itri, p. 210 : «Se vedete, per questo o per altro, fusse utilità o bisogno che frate Raimondo vi venisse, scrivetelo ed egli sarà subito alla vostra obedienza "; et lettre 181 à Nicolas d'Osimo, p. 244 : "Se bisognasse, che per questo o per veruna altra cosa in utilità della santa Chiesa, che frate Raimondo venisse a voi, Padre, scrivetelo : egli sarà sempre obediente a voi ».

23. C. LeE, "Significato dell'autobiografia... ", cit., p. 146. 
Outre de nombreuses lettres, aujourd'hui au nombre de trois cent quatre-vingt trois, on conserve de Catherine vingt-six oraisons et un traité mystique, Il Dialogo della divina Provvidenza: plusieurs centaines de pages au total, dont il ne subsiste que quelques originaux, mais aucun autographe, dans la mesure où cette œuvre a été principalement dictée à des secrétaires, ou simplement transcrite, comme certaines de ses prières, les Orazioni. Ces techniques d'écriture relèvent de pratiques communes au Moyen Âge, où les lettres sont dictées et les prêches retranscrits ${ }^{24}$. Mais ici, elles prennent une dimension toute particulière du fait du statut d'illetterata traditionnellement attribué à l'auteur de cette œuvre.

Tout d'abord, il convient d'établir une distinction dans les modalités de production des différents écrits de Catherine. Si les Orazioni (13761380) ont été "retranscrites, peut-être à son insu, par des disciples " ${ }^{25}$, les autres œuvres relèvent en revanche d'une détermination consciente à produire des écrits voués à circuler. Tel est par définition le cas des lettres, dictées tour à tour à quelque dix-sept secrétaires plus ou moins occasionnels, recrutés au sein de sa famiglia. C'est aussi le cas du Dialogo, produit selon les mêmes modalités, et où Catherine veut " délibérément rassembler et organiser ses intuitions mystiques [...] dispersées dans sa correspondance » à l'intention de ses disciples ${ }^{26}$. Dans ce sens, le Dialogo s'inscrit dans le sillage du Liber, composé entre 1292 et 1296 par Angèle de Foligno, mystique elle aussi, et rattachée au tiers ordre franciscain. Elaboré oralement en langue vulgaire, le Liber est ensuite transcrit en latin par frère Arnaldo qui en supervise la diffusion. Contrairement à la plupart des mystiques ombriennes ou toscanes qui la précèdent qui n'ont pas laissé d'œuvre et dont l'expérience ne nous est parvenue qu'à travers des hagiographies, Angèle de Foligno, sans prendre la plume elle-même, conquiert un véritable statut d'auteur du fait que ses paroles sont immédiatement fixées par écrit ${ }^{27}$.

24. Pour la retranscription des prêches de Bernardin de Sienne par exemple, voir Carlo Delcorno, Exemplum et letteratura tra Medioevo e Rinascimento, Bologne, Il Mulino, 1989, p. 127-162 ; pour la transcription des lettres dans le cadre de l'ars dictandi, voir James J. Murphy, La retorica nel Medioevo, Una storia delle teorie retoriche da S. Agostino al Rinascimento, trad. it. de Vincenzo Lacitra, Naples, Liguori, 1988, p. 223-304 (éd. originale anglaise : 1974).

25. Catherine de Sienne, Les oraisons, trad. Lucienne Portier, Paris, Cerf, 1992, introduction, p. 8.

26. Bernard SEse, Petite vie de Catherine de Sienne, Paris, Desclée de Brouwer, 2000, p. 115.

27. Il est vrai qu'il reste quelques rares textes de mystiques précédant Angèle de Foligno, comme Claire d'Assise (1193-1253), Béatrice I d'Este (1200-1226) et Umiltà de Faenza (1226-1310), toutes trois issues de la noblesse. En revanche, l'expérience spirituelle d'Umiliana Cerchi (1219-1246) et de Marguerite de Cortone (1247-1297), qui n'ont pas produit de textes, passe dans la tradition écrite par le biais de leurs hagiographes respectifs, Vito da Cortona et Giunta Bevegnati. Pour un panorama des mystiques médiévales italiennes, cf. l'anthologie de Giovanni Pozzi et Claudio Leonardi, Scrittrici mistiche italiane, Gênes, Marietti, 1988, p. 61-225. En ce qui concerne la place d'Angèle de Foligno dans le 
C'est bien dans cette tradition que s'inscrit Catherine, qui semble avoir une conscience encore plus aiguë du pouvoir inhérent à la parole écrite. Comme l'a montré Marina Zancan, Catherine dépasse le modèle proposé par Angèle de Foligno, dans la prétention qu'elle affiche de promouvoir elle-même la diffusion de son message, initiative jusque-là exclusivement assumée par les clercs. Avant de mourir, elle écrit en effet à son confesseur, pour désigner elle-même un comité éditorial chargé de rassembler et de diffuser son œuvre :

Anco vi prego che il libro e ogni altra scrittura che trovaste di me, voi e frate Bartolomeo, e frate Tommaso e il Maestro, ve le rechiate per le mani; ; e fatene quello che vedete che sia più onore di Dio, insieme con Missere Tommaso. ${ }^{28}$

Il apparaît donc que Catherine, en signifiant ainsi la teneur de l'héritage qu'elle lègue sciemment à Raymond de Capoue et à quelques autres, se considère désormais bel et bien comme un auteur ${ }^{29}$.

S'agissant d'une correspondance reconstituée « au fil de la cueillette » ${ }^{30}$, il importe dans un second temps de se pencher sur la constitution de ces lettres en recueil. Contrairement à Pétrarque, qui réordonne lui-même ses lettres au sein de véritables recueils d'auteur (Familiares, Seniles, etc.) ${ }^{31}$, Catherine se limite à léguer ses lettres et ses prières à l'état d'écrits isolés, de fragments épars, et non pas à l'état de recueils, le Dialogo étant la seule de ses œuvres véritablement constituée en livre et reconnue comme telle

passage des mystiques de l'oralité à l'écriture, et le modèle qu'elle constitue pour Catherine, ces aspects sont soulignés par Marina ZANCAN, "Le lettere di Caterina da Siena ", in Letteratura italiana, a cura di Alberto Asor Rosa, Le opere, vol. I : Dalle origini al Cinquecento, Turin, Einaudi, 1992, p. 623 : "Caterina probabilmente conosce il Liber di Angela e non c'è dubbio che guarda a lei come ad un modello forte di riferimento, non solo per la complessità e la pregnanza della sua esperienza mistica, ma soprattutto, io credo, per la ridefinizione che essa attua del modello da lei incarnato di santità femminile nel momento in cui ha reso le sue parole autorevoli fissandole in scrittura ".

28. Lettre 373, p. 1194 : "Je vous prie aussi que, le livre et tout autre écrit que vous trouveriez de moi, vous-même, frère Bartholomé, frère Thomas et le Maître, vous les rassembliez entre vos mains ; et faites-en ce qui, à vous et à Messire Thomas, vous semblera le plus en l'honneur de Dieu ".

29. Pour ce qui est de l'identité des autres membres de ce comité éditorial désigné par Catherine dans sa lettre-testament, il peut s'agir de Giovanni Tantucci, maître en théologie («il Maestro »), Tommaso Petra, protonotaire apostolique, Bartolomeo Dominici et Tommaso Caffarini (Eugenio Dupré Theseider, "Un codice inedito dell'epistolario di Santa Caterina da Siena ", in Bullettino dell'Istituto Storico Italiano e Archivio Muratoriano, XI, 1932, p. 31, n. 1). Robert Fawtier quant à lui, reconnaît Tommaso della Fonte, premier confesseur de Catherine, (R. Fawtier - L. Canet, La double expérience..., cit., p. 72).

30. L'expression est empruntée à Marie-Madeleine Fragonard, "Editer une correspondance ", in L'épistolaire au XVI siècle, Cahiers V.L. Saulnier, n 18, 2001, p. 54.

31. Cf. Francesco Petrarca, Lettres familières Livres I-III - Rerum familiarium libri I-III, trad. de André Longpré, introduction et notes d'Ugo Dotti, Paris, Les Belles Lettres, 2002, p. XV : « [Pétrarque] découvrit [...] les lettres de Cicéron à Atticus et celles, moins importantes, à Brutus et à son frère Quintus, et décida donc de donner vie lui-même à son propre corpus épistolaire, en organisant selon des finalités artistiques les lettres qu'il avait jusqu'alors écrites et dont, comme nous l'avons dit, il avait gardé copie". 
par son auteur («il libro e ogni scrittura la quale trovaste di me [...] ve la rechiate per le mani $")^{32}$.

Ce travail de rassemblement des lettres est en fait entrepris par ses disciples avant sa mort, dans un souci de conservation de l'enseignement de leur mère spirituelle. C'est ainsi que se constituent les premiers recueils ensuite utilisés en vue de son procès de canonisation en 1461, avant de servir à l'élaboration de la première édition substantielle qu'en donne Aldo Manuzio en $1500^{33}$, marquant ainsi le point de départ d'une longue tradition éditoriale. Les finalités spécifiques à chacune des phases de la constitution de ce recueil épistolaire, sont à l'origine de diverses altérations, voire de mutilations du texte initial. Aux adjonctions dues aux secrétaires s'ajoutent en effet les coupures et corrections opérées par les éditeurs successifs, de sorte que les lettres que nous lisons aujourd'hui ne reflètent pas au sens strict la parole dictée par Catherine. Ainsi quand on parle de Catherine comme auteur des lettres, il convient d'entendre ce nom dans une sorte de dimension chorale, en étant conscient qu'il s'agit d'une écriture à plusieurs mains, "dans une certaine mesure d'une ouvre collective ", comme l'affirme Carlo Delcorno au sujet des sermons prononcés par Bernardin de Sienne, et transcrits par un autre ${ }^{34}$.

La critique minutieuse à laquelle Robert Fawtier a soumis au début du siècle dernier les œuvres de Catherine, nous autorise cependant à considérer ces lettres comme globalement authentiques ${ }^{35}$. Cet avis est d'ailleurs confirmé ensuite par l'ensemble de la critique qui s'accorde aussi pour dire que les modifications qui auraient pu intervenir en amont de la constitution des recueils, c'est-à-dire au moment même de la dictée des lettres, sont minimes et sporadiques. Cette conviction repose non seulement sur l'autorité dont Catherine jouit auprès de ses secrétaires et disciples, mais aussi sur le fait qu' elle est en mesure de relire cette transcription et donc de

32. Si le matériau de sa correspondance est organisé d'une certaine manière dans une œuvre, c'est justement au sein du Dialogo où certaines de ses lettres antérieures (la 273 par exemple) sont refondues dans un nouveau moule, bien distinct du genre épistolaire. Pour les rapports entre le Dialogo et les lettres, cf. Eugenio Dupré Theseider, "Sulla composizione del Dialogo di santa Caterina da Siena ", in Giornale storico della letteratura italiana, 117, 1941, p. 161-202.

33. Epistole devotissime de sancta Catharina da Siena, in casa de Aldo Manutio, Venetia, 1500 (le matériel de l'édition est rassemblé par le dominicain Bartholommeo da Alzano de Bergame).

34. C. Delcorno, Exemplum..., cit., p. 127 : «il capolavoro di Bernardino è da ravvisare nella serie di prediche tenute sul Campo di Siena dal 15 agosto al 5 ottobre del 1424, raccolte "de verbo ad verbum" dal cimatore di panni Benedetto di maestro Bartolomeo : un testo che risulta da un atto di collaborazione tra oratore e uditore, ed è opera in qualche misura collettiva ; e pure riflette con singolare scrupolo fin anche la fisicità della voce dell'oratore ".

35. R. Fawtier, Sainte Catherine..., cit., p. 335 : «En somme, sauf l'addition à la lettre 90 et peutêtre la lettre 97, la correspondance de sainte Catherine de Sienne paraît bien fournir un document historique authentique et utilisable pour l'histoire de sainte Catherine ». 
la contrôler ${ }^{36}$. Pour ce qui est des ajouts de fin de lettres où les secrétaires expriment en leur nom quelque message personnel à l'attention du destinataire, tout prête à croire qu'ils ne sont donc pas écrits à l'insu de Catherine ${ }^{37}$. Les modifications ultérieures, dues au comité éditorial désigné par Catherine, consistent quant à elles la plupart du temps à supprimer la partie finale des lettres ${ }^{38}$. Consacrées à des questions pratiques et très circonstancielles, ces lignes sont également jugées inutiles dans la perspective d'édification des fidèles qui est celle des premiers éditeurs ${ }^{39}$.

Aussi particulières qu'elles puissent apparaître, les circonstances qui président à la production de ces lettres ne constituent pas, selon Adriano Prosperi, un cas isolé, mais semblent au contraire représentatives de l'écriture mystique au féminin, d'Angèle de Foligno à Maddalena de' Pazzi, dont les textes sont transcrits par des consœurs ${ }^{40}$. Dans ce sens, le cas de Catherine semble emblématique d'une certaine impossibilité, pour la mystique féminine médiévale, d'établir un rapport direct à l'écriture, tant

36. C'est l'avis que partagent par exemple Eugenio Dupré Theseider et Marina Zancan : Epistolario di Santa Caterina, a cura di Eugenio Dupré Theseider, Rome, R. Istituto storico italiano per il Medio Evo, 1940, 1 vol., p. XV-XVI : " parmi non verosimile che essi [i segretari] abbiano volutamente alterato i concetti che mettevano in carta per la Santa, da loro così fervidamente venerata ed ammirata, quale rivelatrice di sovrumane verità »; M. ZANCAN, « Le lettere... », cit., p. 597 : « nel primo passaggio, dalle parole di Caterina alla scrittura del segretario, le eventuali alterazioni del pensiero o delle forme devono essere state minime e del tutto occasionali. Dato il prestigio e l'autorevolezza di cui [Caterina] godeva all'interno della sua comunità, gli estensori delle lettere non potevano infatti, che attenersi con scrupolo e con rispetto al dettato di Caterina la quale del resto, poiché sapeva leggere, era in grado di controllare le trascrizioni del suo pensiero ".

37. Pour se limiter ici à deux exemples, on citera les post-scriptum des lettre 129 et 298, dont les transcriptions sont respectivement dues à la Mantellata Cecca (Francesca di Clemente Gori) et au poète Neri di Landoccio Pagliaresi : lettre 129, p. 1204 : «Alessa e Caterina, e io, Cecca Pazza, vi ci mandiamo molto raccomandando. Gesù, Gesù. Catarina, serva de’servi di Dio inutile. Vi si raccomanda frate Raimondo e frate Tommaso "; lettre 298, p. 914 : « Io Neri del quattrino, che tu sai, ti prego che mi raccomandi a don Jeronimo de'Frati della Rosa, ma non pugnare quanto a frate Simone ».

38. La collation de divers recueils manuscrits des lettres a permis de montrer que certaines lettres initialement rassemblées par les secrétaires dans leur version intégrale, sont coupées dans un second temps, au moment où leur diffusion sort du cercle restreint des disciples pour toucher un public plus étendu. Dans ce nouveau contexte, le contenu de ces fins de lettres apparaît en effet trop concret, et peut-être trop décalé par rapport au ton mystique du corps des missives. Afin de donner une idée du contenu de ces parties coupées, on peut citer la fin de la lettre 248 publiée par E. Dupré Theseider, "Un codice inedito... ", cit., p. 52: "Date a Francesco el libro e’ privilegii, perché vi voglio scrivere alcuna cosa, e 'l privilegio voglio per fare dire la messa si che daretegli. Cento migliaia di volte mi confortate Bartalo e monna Orsa tenerissimamente, e benedicete Bastiano e tutti gli altri figliuoli e figliuole. Yhu dolce, yhu amore ". Pour le texte de ces fins de lettres, cf. E. Dupré Theseider, ibid., p. 52-56, et Bacchisio R. Moтzo, Alcune lettere di Santa Caterina da Siena in parte inedite, Siena, Lazzeri, 1911, p. 11-27.

39. L'édition que Aldo Manuzio donne des lettres (Epistole devotissime...) est introduite par une dédicace à Francesco Piccolomini (neveu du Pape Pie II qui canonise Catherine en 1461), où l'éditeur souligne en effet la finalité de son travail : un message de réforme religieuse et morale adressée à l'Église et à l'Italie. Pour le texte de cette dédicace, cf. M. ZANCAN, « Le lettere... ", cit., p. 601-602.

40. Adriano Prosperi, «Lettere spirituali ", in Donne e fede, Santità e vita religiosa in Italia, a cura di Lucetta Scaraffia e Gabriella Zarri, Rome-Bari, Laterza, 1994, p. 229 : " queste caratteristiche si ritrovano diffusamente nella vicenda della scrittura femminile di lettere e di cose spirituali, tanto da poterli considerare caratteri originali del genere ». 
comme action, puisque Catherine dicte à ses secrétaires, que comme produit résultant de cette action, puisqu'elle confie la diffusion de ses écrits à un comité éditorial.

Cette mise à distance est encore celle qui ressort quand on observe l'illustration du frontispice de l'une des premières éditions catheriniennes : inspirée par le Christ lui-même, Catherine dicte simultanément à trois secrétaires $^{41}$. Si l'on songe à la représentation que le Caravage donne, bien que deux siècles plus tard, de Matthieu écrivant, plume à la main, sous la dictée de l'ange, on peut être frappé par cette différence de rapport entre parole inspirée et écriture ${ }^{42}$. Dans le cas de Matthieu, le rapport à l'écriture est direct, et la médiation opère au niveau de l'inspiration, puisque c'est l'ange qui explique et qui dicte la parole divine. Pour Catherine au contraire, le rapport à la parole divine est direct, puisque c'est le Christ lui-même qui lui inspire ce qu'elle dit. La médiation est en revanche décalée dans le passage à l'écriture, puisqu'elle n'écrit pas elle-même comme Matthieu, mais dicte à des secrétaires. La présence de secrétaires écrivant sous la dictée dépasse ici le simple cadre des usages épistolaires précédents selon lequel les lettres sont systématiquement dictées. Dans cette représentation, qui illustre d'ailleurs la rédaction du Dialogo, la position des secrétaires, assis, écrivant sous la dictée d'une femme, souligne l'autorité dont Catherine jouit auprès de ses disciples, mais rappelle aussi son statut d'illettrée ${ }^{43}$.

Que faut-il entendre par ce terme d' « illetterata " ? Que Catherine est incapable de tenir une plume, c'est-à-dire analphabète, ou simplement qu'elle n'est pas en mesure d'écrire en latin, bien que sachant le lire ${ }^{44}$ ? C'est une question à laquelle la réponse n'a cessé d'évoluer, sans doute justement à la faveur de cette polyvalence du terme.

Dans ses Miracoli, l'Anonimo Fiorentino nous donne l'image de Catherine se consacrant à la lecture de libri santi ${ }^{45}$. Son hagiographe

41. Santa Caterina da Siena, Dialogo, Venezia, L. De Soardi, 1504.

42. Saint Matthieu et l'Ange, conservé à l'église de Saint-Louis-des-Français à Rome.

43. S'agissant de la rédaction du Dialogo, l'impossibilité d'écrire pourrait aussi signifier l'état d'extase traditionnellement associé à la composition de cette œuvre en particulier.

44. La définition de l'illettrisme au Moyen Âge est assez étendue, comme l'explique Mariateresa Fumagalli Beonio Brocchieri, "L'intellectuel ", in L'homme médiéval..., cit., p. 203 : " Le terme d'“illettré" avait aussi un sens très large, il comprenait qui ne savait ni lire ni écrire (ceux que nous appelons analphabètes), mais aussi d'autres personnes, ceux qui ne connaissaient pas le latin, la langue par excellence ou qui le savaient à peine [...] ou bien qui ne savaient pas l'écrire mais le comprenaient un peu ".

45. Anonimo Fiorentino, Questi sono émiracoli della B. Caterina, Appendice a SANTA Caterina DA Siena, Le Lettere, Siena, Misciattelli, 1921, vol. VI, p. 190 : "Tutto l'altro tempo del dì, poich'è levata, ispende o in ammaestrare genti di seguire la via di Dio, o in contemplare, et di stare rapita come di sopra è detto, o in leggere libri santi ». L'expression « libri santi » utilisée par l'Anonimo Fiorentino, contem 
Raymond de Capoue, confirme en effet qu'elle sait lire et accède donc aux textes latins, sans être cependant en mesure de s'exprimer dans cette langue. À ce sujet, Raymond de Capoue évoque les conditions miraculeuses dans lesquelles Catherine aurait appris à lire, précisant que ce miracle serait intervenu après des efforts soutenus d'alphabétisation ${ }^{46}$.

Aujourd'hui, la critique semble s'accorder pour dire que Catherine lit péniblement et qu'elle n'a pas directement accès à toutes les sources religieuses qui transparaissent pourtant dans ses lettres. C'est donc surtout par le biais de la tradition orale que Catherine assimile cette culture, que ce soit par l'intermédiaire de la prédication ou de la liturgie, ou à travers les rapports intenses et continus qu'elle entretient avec certains des membres les plus instruits de sa communauté ${ }^{47}$. Quant à savoir si Catherine sait écrire, cette hypothèse semble corroborée par Catherine elle-même à la fin de l'une de ses lettres, la lettre 272 adressée en 1377 à Raymond de Capoue où elle dit prendre la plume pour la première fois après avoir reçu miraculeusement le don de l'écriture :

Questa lettera e un' altra che io vi mandai, ho scritte di mia mano in su l'Isola della Rocca, con molti sospiri e lacrime ; in tanto che l'occhio, vedendo, non vedeva ; ma piena di ammirazione ero di me medesima, e della bontà di Dio, considerando la sua misericordia verso le creature che hanno in se ragione, e la sua Providenzia ; la quale abondava verso di me, che per refrigerio, essendo privata della consolazione, la quale per mia ignoranzia non cognobbi, maveva dato, e proveduto con darmi l'attitudine dello scrivere, acciocché discendendo dall'altezza, avessi un poco con chi sfogare il cuore, perché non scoppiasse. ${ }^{48}$

porain de Catherine, ne désigne cependant pas nécessairement les Saintes Écritures, mais peut aussi se référer à quelque récit hagiographique, comme le suggère un passage immédiatement précédent des Miracoli : "ella legge delle cose dei Santi ».

46. Raymond De Capoue, Vie de sainte Catherine..., cit., p. 114-115 : «Et puisque nous parlons ici de la psalmodie, je dois vous dire, lecteur, que cette vierge savait lire, sans l'avoir appris d'aucun homme de ce monde. Je dis qu'elle savait lire et non pas parler latin, mais simplement lire et prononcer les syllabes. Elle me raconta à ce sujet, que, voulant réciter les louanges divines et les heures canoniques, elle avait résolu d'apprendre à lire. S'étant fait écrire un alphabet, elle l'étudiait avec le secours d'une compagne. Après avoir travaillé pendant plusieurs semaines sans parvenir à l'apprendre, elle imagina d'avoir recours à la grâce de Dieu, pour éviter cette perte de temps ».

47. Giuliana Cavallini, "Fonti neotestamentarie degli scritti cateriniani ", in Atti del Congresso internazionale di studi cateriniani, Rome, Curia Generalizia O.P., 1981, p. 48 : « sembra legittimo pensare che i principali veicoli siano stati la predicazione et la liturgia ». M. ZANCAN, "Le lettere... », cit., p. 616 : « Mi sembra tuttavia ragionevole pensare che la transmissione di queste fonti debba essere in questo caso avvenuta in gran parte all'interno di una tradizione orale ".

48. Lettre 272, p. 1164 : «Cette lettre et une autre que je vous ai envoyée, je les ai écrites de ma main à l'île de la Rocca avec beaucoup de soupirs et de larmes, à tel point que l'œil en voyant ne pouvait plus voir ; mais j'étais pleine d'admiration envers moi-même et envers la bonté de Dieu, considérant sa miséricorde envers les créatures douées de raison, et sa Providence qui abondait envers moi, car, pour me soulager, moi qui suis privée de la consolation que, par mon ignorance, je n'ai pas connue, il m’avait fait un don et pourvue de ce soulagement, en me donnant la faculté d'écrire, afin que, descendant des hauteurs, je puisse un peu soulager mon cœur auprès de quelqu'un pour qu'il n'éclate pas ». 
Partant de la constatation qu'un miracle d'une telle ampleur n'est pas mentionné dans l'hagiographie de Raymond de Capoue, Robert Fawtier soumet la lettre 272 à une critique minutieuse à l'issue de laquelle il rejette l'authenticité, non de toute la lettre, mais du post-scriptum cité, ajouté selon lui par Tommaso Caffarini en vue du procès de canonisation de Catherine ${ }^{49}$.

Marina Zancan, considérant au contraire ce passage comme authentique, fait de ce moment décisif de l'accès personnel et officiel de Catherine à la parole écrite, un point charnière dans son évolution vers une prise d'autonomie dans le domaine de l'écriture qui va bien au-delà de la simple désignation d'un comité éditorial chargé de diffuser son ouvre. Dans ce sens, ce passage à l'écriture illustrerait cette phase de transition générale dans la production épistolaire, au moment où l'on glisse des lettres dictées, caractéristiques du Moyen Âge, vers l'élaboration de lettres autographes telles qu'elles fleurissent à la Renaissance.

Selon Marina Zancan, cette prise d'autonomie n'intervient qu'en 1377, alors que Catherine dicte ses lettres depuis plus de dix ans, justement parce qu'auparavant, elle ne jouit peut-être pas d'une autorité suffisante pour imposer cette dimension nouvelle de l'écriture dans le modèle de sainteté féminine, sans risquer d'être suspectée ${ }^{50}$. Il suffit en effet de se rappeler les soupçons qui conduisent à sa convocation, en 1374, au chapitre général des dominicains où son orthodoxie fait l'objet d'une enquête.

En vérité, par-delà la question de l'authenticité de la lettre 272 et de l'accès direct de Catherine à l'écriture, il ressort cependant que le rapport de la femme à la parole écrite a encore, au XIve siècle, quelque chose de problématique. Dans ce climat de troubles, l'incapacité du clergé à se réformer apparaît comme une trahison des clercs à leur mission pastorale. Face à cette défaillance du clergé, des laïques illettrées, comme Brigitte de Suède ou Catherine, se sentent investies d'une "fonction de suppléance " typiquement prophétique, pour reprendre les termes d'André Vauchez ${ }^{51}$.

49. R. Fawtier, Sainte Catherine de Sienne..., cit., p. 326 : "Quant au post-scriptum, on voit très bien comment Caffarini a été amené à l'ajouter. Il s'était décidé à établir que Catherine avait non seulement appris à lire miraculeusement comme le disait Raymond de Capoue, mais aussi à écrire comme ne le disait pas la Légende Majeure ».

50. M. ZANCAN, "Le lettere... ", cit., p. 596.

51. André Vauchez, Saints, prophètes et visionnaires, le pouvoir surnaturel au Moyen Âge, Paris, Albin Michel, 1999, p. 128 : "Dieu avait choisi cette lä̈que inculte [Brigitte de Suède] à cause de la trahison des clercs, auxquels il avait confié la responsabilité des âmes mais qui étaient devenus infidèles à leur mission. [...] On en revient en quelque sorte, deux siècles plus tard, à l'eschatologie judiciaire qui caractérisait déjà l'œuvre d'Hildegarde de Bingen, et l'affirmation de la fonction de suppléance exercée par le prophète". 
Déjà revendiquée avant elles par Hildegarde de Bingen, cette fonction de suppléance ne consiste pas à remettre en cause le rôle de la hiérarchie ecclésiastique, mais à instiguer la réforme nécessaire à l'accomplissement de sa mission. Cette logique, selon laquelle la parole divine, faute d'être entendue par les clercs lettrés est révélée à d'humbles laïcs illettrés, renvoie à la conception évangélique, selon laquelle le mystère divin " caché aux sages et aux intelligents est révélé aux tout-petits " (Matthieu 11, 25). C'est donc bien dans cette perspective que s'inscrit la désignation de femmes, laïques et illettrées, comme porte-parole de Dieu ${ }^{52}$. Dans ce sens, l'intervention des secrétaires, intermédiaires cultivés, traduit en quelque sorte le statut d'illettrée qui est traditionnellement lié à l'élection divine dont Catherine se réclame pour prendre la parole.

À travers cette première approche des textes catheriniens sous l'angle à la fois socio-historique et philologique, il apparaît donc que la présence de médiateurs lettrés à travers qui Catherine consolide en aval l'autorité de sa parole dans le domaine de l'écriture, est en fait intimement liée à l'autorité divine dont elle se réclame en amont, et qui légitime sa prise de parole.

\section{Inspiration divine et auctoritas}

C'est à travers une approche littéraire cette fois, que l'on parvient à cerner cet autre type d'autorité sur lequel se fonde en amont la force de persuasion du discours catherinien : d'une part une autorité supérieure et transcendante, qui est le propre de l'inspiration mystique, et d'autre part la référence aux Écritures qui sont l'auctoritas par excellence de la rhétorique sacrée : «Exclues du ministère de la parole au sein de l'Église, [les femmes et surtout les laïques] s'en emparent en se réclamant d'une élection divine ", explique André Vauchez au sujet des mystiques visionnaires de la fin du Moyen $\hat{A g e}^{53}$. On retrouve en effet cette revendication, également commune à d'autres prophètes, chez Catherine qui, à plusieurs reprises dans les lettres aux papes, se présente comme le porte-parole de Dieu dont elle rapporte les messages selon différentes modalités : au style direct, ou

52. C. Lee, "Significato dell'autobiografia... ", cit., p. 808 : "In primo luogo la donna si sente in obbligo di dichiarare la sua ignoranza e incapacità nello scrivere e dunque il suo bisogno di aiuto. Ildegarda di Bingen, per esempio era attenta a distinguere la luce e la voce divine che che ispiravano $\mathrm{i}$ suoi scritti dalla sua "paupercula feminea forma", cioè dalla sua misera forma femminile. Angela da Foligno e Caterina da Siena, che non avevano dietro di sé un'educazione conventuale, si dichiararono analfabete, anche se sembra che sapessero leggere ".

53. A. Vauchez, "Le saint... ", cit., p. 363. 
bien seulement en substance, ou encore à travers le regard qu' elle porte sur le sens de l'histoire.

Le cas le plus transparent de renvoi à la parole divine est celui où Catherine rapporte au style direct des propos échangés avec le Christ. On en trouve un seul exemple dans les lettres aux papes; il s'agit d'un passage de la lettre 238, adressée à Grégoire XI en 1376, et constitué en fait de deux répliques présentées comme l'extrait décisif d'un dialogue plus développé :

[...] pregando io il nostro dolce Salvatore per voi, siccome mi mandaste dicendo, manifestando egli, ch'io dicessi a voi che voi doveste andare, e io scusando, reputandomi indegna d'essere annunziatrice di tanto misterio, dicevo : « Signore mio, io ti prego che se egli è la tua volontà che egli vada, che tu gli accresca e accenda di più il desiderio suo ». Diceva, per la sua bontà, il nostro Salvatore : « Digli sicuramente che questo ottimo segno gli do, che ella è la mia volontà che egli vada : ché quanto più contrari gli verranno, e più gli sarà contraddetto che egli non vada, più si sentirà crescere in sé una fortezza, che uomo non parerà che gli la possa tollere; che è questo contra 'l modo suo naturale. Or ti dico, ch'io voglio che egli levi la Croce santissima sopra gl'infedeli ; e levila sopra de' sudditi suoi, ciò sono quelli che si pascono e notricansi nel giardino della santa Chiesa che sono ministratori del sangue mio. Dico che sopra costoro voglio che egli levi la Croce ; cioè in perseguitare e' vizi e i difetti loro. Divelto il vizzio, è piantata la virtù, ponendo questa Croce in mano di buoni pastori e rettori nella Santa Chiesa. E se non ci è di fatti, vuole che quelli che sono a fare, voi miriate che siano buoni e virtuosi che non temano la morte del corpo loro. Non vuole Dio che si ragguardi agli stati e alle grandezze e alle pompe del mondo, perocché Cristo non ha conformità con loro; ma solo alla grandezza e richezza della virtù. A questo modo li buoni con l'affetto della croce perseguiteranno li vizii delli cattivi. ${ }^{54}$

Ce passage est intéressant à plus d'un titre. Tout d'abord, parce que la forme dialoguée utilisée ici par Catherine, anticipe d'un an, bien que de manière encore embryonnaire, la structure qu'elle utilisera dans son Dialogo pour exprimer sa doctrine. D'autre part, ce passage fait apparaitre, dans la bouche du Christ lui-même, trois des quatre grands thèmes du

54. Lettre 238, p. $88:$ « [...] alors que je priais pour vous notre doux Sauveur, ainsi que vous me l'aviez demandé, et comme il manifestait sa volonté que je vous dise que vous deviez partir, moi, le priant de m'excuser car je me considérais indigne d'être l'annonciatrice d'un tel mystère, je disais : "Mon Seigneur, je te prie, si c'est ta volonté qu'il parte, de faire grandir et d'enflammer davantage son désir ". Dans sa bonté, notre doux Sauveur répondait : « dis-lui avec assurance que je lui donne cet excellent signe pour lui prouver que ma volonté est qu'il parte : plus il rencontrera d'obstacles, et plus on s'opposera à lui pour qu'il ne parte pas, plus il sentira grandir en lui une force telle qu'aucun homme ne semblera pouvoir la lui enlever, ce qui est contraire à sa nature. Je te dis que je veux qu'il lève la très sainte Croix contre les Infidèles et qu'il la lève contre certains de ses sujets, à savoir ceux qui trouvent pâture et se nourrissent dans le jardin de la sainte Église, ceux qui administrent mon sang. Je dis que sur ceux-ci je veux qu'il lève la Croix, et qu'il le fasse en pourchassant les vices et les défauts de ceux-ci. Une fois le vice arraché, la vertu est plantée en plaçant cette Croix entre les mains des bons pasteurs et bons recteurs de la sainte Église. Et s'il n'y en a pas déjà, il veut que, pour ceux qui doivent être nommés, vous veilliez à ce qu'ils soient bons et vertueux, et qu'ils ne craignent pas la mort de leur corps. Dieu ne veut pas que l'on considère les titres, les grandeurs et les pompes de ce monde, parce que le Christ n'est pas fait à cette image, mais seulement à l'image de la grandeur et de la richesse de la vertu. De cette manière, les bons, avec l'amour de la Croix, pourchasseront les vices des mauvais ». 
discours de persuasion que Catherine adresse à Grégoire XI : le retour à Rome, la croisade et la réforme de l'Église. La continuité alors établie entre la parole divine et le discours de Catherine se répercute en fait jusque dans la forme même de l'expression. Non seulement les formules utilisées ici sont le calque de celles que Catherine emploie en son propre nom dans d'autres lettres ${ }^{55}$, mais la fin de la réplique du Christ montre que l'on glisse, de manière insensible, du style direct au style indirect (d'une manière d'ailleurs si insensible que les éditeurs successifs ne referment les guillemets qu'après le mot " cattivi ", alors que la réplique semble s'arrêter à "Chiesa ", comme le suggère le passage à la troisième personne dans la phrase suivante ("E se non ci è di fatti, vuole che ») ${ }^{56}$. Ce bref échange de répliques montre enfin que Catherine se sent dans l'obligation de souligner l'inadéquation de son statut à la teneur du message qu'il lui incombe de rapporter, et dont elle doit convaincre le Pape ("reputandomi indegna d'essere annunziatrice di tale misterio »).

On remarque d'ailleurs que, selon un schéma classique de prétérition, Catherine ne semble souligner cette inadéquation que pour mieux mettre ensuite en valeur sa compensation à travers l'excellent signe envoyé au Pape (" digli sicuramente che questo ottimo segno gli do »). C'est d'ailleurs sur l'excellence de ce signe que semble devoir reposer toute l'assurance de Catherine, en dépit de son indigne statut.

Mais ce passage au style direct ne représente qu'une partie du dialogue dont le début et la fin sont rapportés en substance (" sembra che la bontà di Dio tre cose vi richiegga " ${ }^{57}$, ou comme on l'a vu "vuole che », et plus loin " non vuole Dio che si ragguardi agli stati»). En vérité, l'absence du verbe « dire " dans ces différents exemples, n'autorise pas à parler exactement de style indirect. Tout se passe plutôt comme si Catherine ne rapportait qu'en substance le message divin en le reformulant avec ses propres mots. C'est aussi le cas de la lettre 233 où Catherine apparaît véritablement comme une visionnaire consultée par Grégoire XI :

Dissemi Padre Raimondo, per parte vostra, ch'io pregasse Dio, se doveste avere impedimento : e io già n'avea pregato, innanzi e dopo la comunione santa, e non vedea né morte né pericolo neuno. 58

55. À titre d'exemple, on pourrait citer l'image du jardin de l'Église dont il faut arracher les mauvaises herbes pour y planter des herbes odoriférantes, et que l'on retrouve, en des termes similaires, dans les lettres 185, 206, 218, 252, 255 et 270, pour ne parler que des lettres à Grégoire XI.

56. Cf. l'édition de Meattini citée, mais aussi celle de Tommaseo (CATERINA DA SiEnA, Le lettere..., cit., p. 311) et celle de Lodovico Ferretti (Lettere di S. Caterina da Siena, vergine domenicana, con note di Mons. Lodovico Ferretti, 5 vol., Siena, Tip. S. Caterina, 1918-1930, vol. III, p. 438).

57. Lettre 238, p. 88 : "Il semble que la bonté de Dieu vous demande trois choses ".

58. Lettre 233, p. 86 : «Le père Raymond m'a demandé de votre part de prier Dieu pour savoir si vous deviez rencontrer des obstacles, mais j'avais déjà prié Dieu dans ce sens, avant et après la sainte communion, et je ne voyais ni mort ni danger d'aucune sorte ». 
Mais il s'agit là aussi d'un exemple isolé. La plupart du temps en effet, c'est plutôt à travers son regard prophétique, c'est-à-dire la signification spirituelle qu'elle dégage des événements que Catherine révèle les intentions de Dieu. Elle décrypte ainsi les malheurs de l'époque (peste, guerre, crise économique et schisme) comme des fléaux envoyés par Dieu aux chrétiens pour les punir de leurs manquements, ainsi que l'on peut le voir dans la lettre 185:

Qui ho inteso che avete fatto i Cardinali. Credo che sarebbe onore di Dio, e meglio di noi, che attendeste sempre di fare uomini virtuosi. Se si farà il contrario, sarà grande vituperio di Dio, e guastamento della Santa Chiesa. Non ci maravigliamo poi, se Dio ci manda le discipline e i flagelli suoi ; perocché giusta cosa è. ${ }^{59}$

Cet exemple illustre la conception providentielle que Catherine a de l'histoire, et selon laquelle l'origine des problèmes rencontrés par les États pontificaux a une double dimension : une dimension immanente, la responsabilité humaine, à savoir le manquement du pape à son devoir de nommer de bons cardinaux, et une dimension transcendante, le châtiment de Dieu, c'est-à-dire l'interprétation des évènements comme le bras armé de la justice divine. En vérité, plus que parallèles, ces deux explications apparaissent plutôt emboîtées l'une dans l'autre : en effet, c'est parce qu'il manque à son devoir que Grégoire XI risque de déclencher la colère divine et d'attirer sur la chrétienté les fléaux envoyés par Dieu. Ainsi la dimension divine et dimension humaine se répondent-elles dans une vision de l'histoire orientée dans le sens de l'amélioration de l'homme. Loin d'être en concurrence, ces deux dimensions des tribulations de l'Église s'articulent en effet dans l'analyse de Catherine. Pour elle, ce sont les manquements réitérés des chrétiens à leur mission, qui provoquent la colère de Dieu et qui attirent ses châtiments. À quelque niveau que ce soit, humain ou divin, il apparaît donc que l'origine des maux de l'Église n'est pas à rechercher à l'extérieur de celle-ci, mais en son sein même. Cela explique la requête obsédante elle aussi que Catherine adresse au Pape sur tous les tons, passant de celui du conseil à celui de l'exhortation prophétique : celle de la réforme de l'Église. C'est bien à la prophétesse " qu'incombe la charge redoutable de convaincre les hommes que la colère de Dieu peut encore être apaisée avant le châtiment suprême qui ne tardera pas à s'abattre sur eux ", explique André Vauchez rappelant qu'avec le courant visionnaire féminin de la fin du XIV et du Xve siècle, on abandonne le

59. Lettre 185 , p. 63 : «J'ai entendu dire ici que vous avez nommé les cardinaux. Je crois qu'il en irait de l'honneur de Dieu, et plus encore du nôtre, que vous preniez toujours soin de nommer des hommes vertueux. Si on fait le contraire, ce sera injurier grandement Dieu et dévaster la sainte Église. Ne nous étonnons pas ensuite si Dieu nous envoie ses châtiments et ses fléaux, parce que ce n’est que justice ». 
prophétisme exégétique prédominant en Occident depuis Joachim de Flore, pour revenir à un message « beaucoup plus moralisant, axé sur la colère de Dieu ${ }^{60}$.

Il apparaît donc que le discours de persuasion que Catherine adresse au Pape trouve aussi une légitimation dans l'élection divine dont elle se réclame en se faisant l'oracle de Dieu. Mais comme on l'a vu, elle ne rapporte que très rarement au Pape des échanges ou des messages précis, comme elle le fait au contraire dans son Dialogo. Dans les lettres aux Papes en effet, cette élection semble plutôt se traduire par une faculté à décrypter le sens des événements selon une logique transcendante.

Une des modalités de cette lecture des événements est liée à l'autre source d'autorité sur laquelle Catherine fonde, en amont, son discours de persuasion : les Écritures, véritable auctoritas mise en avant dans les lettres. À un premier niveau, Catherine utilise la Bible pour éclairer le Pape sur une situation particulière. Tel est par exemple le cas, lorsqu'elle évoque la rébellion de certaines villes des États du pape. Après avoir condamné cette révolte, elle cherche à en expliquer les causes, en dénonçant les excès des légats pontificaux. Elle explique alors que ces villes ont fini par s'opposer au Pape presque malgré elles, comme Pilate condamnant le Christ malgré lui :

Perocché, sentendo il puzzo della vita di molti rettori, e' quali sapete che sono demoni incarnati, vennero in tanto pessimo timore, che fecero come Pilato, il quale per non perdere la signoria, uccise Christo : e così fecero essi che, per non perdere lo stato, vi hanno perseguitato. ${ }^{61}$

Cette comparaison permet à Catherine de rendre compte de la position des rebelles, mais aussi de la nuancer. Comme Pilate qui, malgré sa conviction que le Christ est accusé par jalousie, le fait crucifier pour satisfaire aux grands prêtres, aux anciens et à la foule afin d'éviter le tumulte, les rebelles apparaissent pris dans une contradiction. "A tutti in comune loro pare avere male fatto " explique d'ailleurs Catherine un peu plus haut ${ }^{62}$. Ce passage montre bien comment elle utilise l'Évangile pour décrypter une situation politique, c'est-à-dire pour proposer une certaine lecture de la situation. Il en va de même lorsque, pour convaincre Grégoire XI de faire la paix avec les villes rebelles (c'est-à-dire de lever l'interdit qu'il fait peser

60. A. Vauchez, Saints, prophètes et visionnaires..., cit., p. 128 et 127.

61. Lettre 196, p. 66 : "C'est pourquoi, sentant la puanteur de la vie de nombreux recteurs, dont vous savez qu'ils sont des démons incarnés, ils furent réduits à une telle crainte qu'ils firent comme Pilate qui, pour ne pas perdre le pouvoir, tua le Christ : c'est ce que firent ceux-ci qui, pour ne pas perdre leur importance, vous ont persécuté ".

62. Lettre 196, p. 66 : "à tous, il semble communément avoir mal agi ». 
sur elles), elle recourt à l'image de la brebis égarée : « le vostre pecorelle, le quali come ribelle si sono partite dall'ovile della Santa Chiesa, non obbidienti né subietti a voi padre " ${ }^{63}$. À travers cet exemple, on voit comment Catherine essaie, là encore, de nuancer la situation des rebelles en mettant l'accent sur leur appartenance à l'Église, temporairement mise à mal. En effet, le rapprochement avec la parabole néotestamentaire de la brebis égarée (puis ramenée au bercail) suggère que l'épisode de la rébellion n'est pas une situation définitive, mais plutôt une parenthèse dans une histoire vouée à redevenir harmonieuse. Là aussi, la référence scripturaire permet à Catherine d'imposer une certaine lecture de la situation politique, en mettant l'accent sur la vocation des villes rebelles à faire la paix avec le Pape.

Cet exemple montre bien comment cette première fonction du renvoi biblique, comme décryptage de la réalité, se rattache à la deuxième finalité de la référence scripturaire chez Catherine, à savoir guider le Pape dans sa conduite. Dans cet exemple en effet, l'association entre les rebelles et la brebis égarée amène Catherine à assimiler le Pape au bon pasteur prêt à donner sa vie pour récupérer sa brebis : «Allora goderete [...] perché averete rimessa la pecorella smarrita nell'ovile della Santa Chiesa " ${ }^{64}$. On voit ici que la parabole est utilisée par Catherine pour guider le Pape dans une action au sens propre : lever l'interdit sur les villes rebelles. Quand Catherine souhaite conseiller le Pape sur le discours à tenir, c'est-à-dire sur ses paroles et non sur ses actions, elle a plutôt tendance à recourir aux paroles du Christ.

Tel est par exemple le cas lorsqu'elle essaie de convaincre le pape de rentrer à Rome contre l'avis de ses cardinaux qui disent craindre pour sa vie :

[...] dite a loro arditamente, come disse Cristo a San Pietro quando per tenerezza il voleva ritrarre, che non andasse alla passione; Cristo si rivolse a lui, dicendo : « Va' di po' me, Satanas. Tu mi se' scandalo, cercando le cose che sono dagli uomini, e non quelle che sono da Dio. E non vuogli tu che io compia la volontà del Padre mio ? ". Così fate voi, dolcissimo Padre. ${ }^{65}$

63. Lettre 196, p. 65 : «vos brebis qui, en tant que rebelles, ont quitté le bercail de la sainte Église, refusant de vous obéir et de se soumettre à vous, Père».

64. Lettre 196, p. 66 : «Alors vous vous réjouirez [...] parce que vous aurez ramené la brebis égarée dans le bercail de la sainte Église ».

65. Lettre 233, p. 85-86 : « dites-leur, avec ardeur, ce que le Christ dit à Pierre quand, par tendresse, ce dernier voulait le retenir d'aller à la passion : " "Retire-toi ! Derrière moi, Satan! tu es pour moi une occasion de chute, car tes vues ne sont pas celles de Dieu, mais celles des hommes". Faites-en de même, très doux Père ». Ce passage fait écho à Matthieu 16, 23 selon la Vulgate : "vade post me, Satana! scandalum est mihi, quia non sapis ea, quae Dei sunt, sed ea, quae hominum ”. 
On remarque cependant ici que, par-delà la parole, c'est aussi une attitude, voire franchement une action qui est suggérée au Pape. Mais c'est surtout à travers certaines métaphores bibliques que Catherine rappelle au Pape ses devoirs. Ce n'est pas un hasard si les lettres à Grégoire XI sont littéralement tissées de renvois à l'image du berger, du gardien et de ses clefs, de la pierre ferme et stable. Ces trois images illustrent en effet les fonctions principales du pape : responsable de l'ensemble des chrétiens, détenteur du pouvoir d'excommunier ou de lever l'excommunication, et investi de l'autorité suprême sur laquelle repose l'Église. En tant que tel, le pape apparaît dans les lettres comme le successeur de Pierre ("vicario di Pietro " ${ }^{66}$ ) et donc comme le représentant du Christ sur terre ${ }^{67}$. A travers ces images du berger, du gardien et de la pierre ferme, Catherine, à la manière des prophètes, renvoie donc Grégoire XI à sa mission première de pape. Qu'il s'agisse donc d'imposer une certaine lecture des événements ou d'indiquer au Pape une conduite à tenir, les références scripturaires sont utilisées par Catherine dans le but de persuader son destinataire, en fondant ses conseils sur l'autorité de la parole biblique.

Catherine recourt ainsi à plusieurs passages des Écritures, qu'il s'agisse de personnages bibliques servant d'exempla (ou de "contre-exempla", comme Pilate), d'épisodes entiers ou de paraboles. Comme l'a montré Giuliana Cavallini, il s'agit rarement de renvois à l'Ancien Testament, mais plutôt d'évocations des Évangiles et des épîtres pauliniennes ${ }^{68}$. Si ces références scripturaires sont constantes dans l'écriture catherinienne, elles ne sont presque jamais présentées comme des citations à proprement parler. Dans les lettres aux Papes en effet, on ne trouve qu'un seul exemple de renvoi explicite, dans la lettre 239 : " come dice il nostro Salvatore nel suo Santo Vangelio " ${ }^{69}$. Mais la plupart du temps, aucune formule de ce type ne vient marquer la distance entre la parole catherinienne et l'auctoritas biblique à laquelle elle se réfere. Il s'agit alors d'un type de renvoi plus subtil, presque d'un simple écho, d'une réminiscence biblique au plan lexical concernant une expression ou un mot, voire le sens spécifique donné à un mot. C'est ce que Alvaro Grion appelle les « riecheggiamenti " ${ }^{70}$. Ainsi l'expression utilisée à deux reprises pour introduire une lettre fait-elle écho à une formulation biblique. En fin de souscription en effet, au moment d'exprimer la motivation de sa lettre, Catherine modifie sa formule habi-

66. Lettre 206, p. 70 : "del glorioso pastore Santo Pietro, di cui vicario sete rimasto ».

67. Lettre 218, p. 76 : « imparando dal Padre, Cristo, il cui luogo voi tenete ».

68. G. Cavallini, "Fonti neotestamentarie... ", cit., p. 45.

69. Lettre 239, p. 90 : « comme le dit notre Sauveur dans son saint Évangile».

70. Alvaro Grion, Santa Caterina da Siena. Dottrina e fonti, Brescia, Morcelliana, 1953. 
tuelle ( Scrivo a voi con desiderio di vedervi " ${ }^{71}$ ) pour employer une expression directement inspirée de Luc 22,15 : " con desiderio che io ho desiderato $"{ }^{72}$, et $"$ che io lungo tempo desiderato ${ }^{73}$. Il en va de même pour le sens qu'elle donne au verbe " trarre ", qui semble être, selon Giuliana Cavallini ${ }^{74}$, une réminiscence de Jean 6,44 quand, dans la lettre 196, elle rappelle à Grégoire XI : " per neuno modo si traie tanto il cuore dell'uomo quanto per amore " ${ }^{75}$. Ici en effet, le verbe " trarre » est employé par Catherine au sens biblique du terme, c'est-à-dire pour désigner l'attraction irrésistible exercée par Dieu sur l'homme.

Ces exemples font apparaittre à quel point les références scripturaires se fondent dans la prose de Catherine, et on pourrait se demander si, dans ces conditions, il est encore possible de parler d'auctoritas. En effet, dans le cas des simples échos bibliques, la source n'est pas mise explicitement à distance pour faire clairement apparaître l'auctoritas sacrée sur laquelle se fonde la démarche de persuasion. On peut cependant penser qu'aux yeux d'un lecteur averti, tel que le Pape, ce type de renvoi ne devait pas manquer d'apparaître dans toute sa transparence, sans perdre par-là même la force persuasive propre à une auctoritas.

Pour mener une œuvre de persuasion auprès du pape à propos de questions politiques d'envergure sur lesquelles cette simple laïque n'a aucune autorité, Catherine se présente comme le porte-parole de Dieu, qu'elle en rapporte les messages, ou qu'elle décrypte, à travers son regard prophétique, les évènements de l'époque selon une dimension transcendante. Or si elle a été choisie par Dieu, comme Brigitte de Suède avant elle, c'est aussi en raison de son statut, afin que la vérité élémentaire, qui n'est plus entendue par les clercs, s'exprime par la bouche d'une simple femme. Dès lors, elle ne peut revendiquer à la fois une élection divine et l'accès direct à l'écriture. Dans ce sens, les intermédiaires masculins, des secrétaires aux éditeurs, qui s'interposent constamment entre elle et ses textes, confirment par leur présence le statut d'illettrée lié à l'élection divine de Catherine, tandis que son confesseur dominicain garantit l'orthodoxie de ses propos, et que les disciples rassemblés autour d'elle témoignent de ses dons charismatiques. Il apparaît donc que les différentes sources d'auto-

71. "Je vous écris dans le désir de voir en vous".

72. Lettre 206, p. 68 : "dans le désir que j’ai désiré ».

73. Lettre 270, p. 104 " dans le désir que j’ai longtemps désiré »; cf. Luc 22, 15 : « desiderio desideravi hoc pascha manducare vobiscum, antequam patiar ".

74. G. Cavallini, «Fonti neotestamentarie... ", cit., p. 55.

75. Lettre 196, p. 64 : " on n'attire jamais le cour de l'homme autant que par l'amour "; cf. Jean 6, 44: «nemo potest venire ad me, nisi Pater, qui misit me, traxerit eum ». 
rité extra-textuelles sur lesquelles Catherine fonde sa démarche de persuasion sont intimement liées entre elles. Au niveau textuel, elle consolide cette démarche en invoquant l'autorité de la parole de Dieu à travers cette auctoritas de la rhétorique sacrée que sont par excellence les Écritures, et plus particulièrement les Évangiles, s'inscrivant par-là même dans le sillage des Pères de l'Église, mais aussi de la prédication articulée autour de l'exemplum, telle qu'on la pratique alors au sein des ordres mendiants. 